\title{
AVALIAÇÃO DAS TENSÕES ÀS ESTRUTURAS DE SUPORTE, POR MEIO DO MÉTODO FOTOELÁSTICO, DECORRENTES DAS FORÇAS APLICADAS SOBRE OVERDENTURES RETIDAS EM IMPLANTES COM SISTEMA DE ENCAIXE BARRA-CLIPE
}

\author{
APPRAISAL, BY PHOTOELASTIC METHOD, OF LOAD TRANSMISSION ON \\ SUPPORT STRUCTURES WITH OVERDENTURES RETAINED IN IMPLANTS \\ WITH BAR-CLIP SYSTEM.
}

\author{
Mônica Nogueira Pigozzo* \\ Dalva Cruz Laganá* \\ Pedro Yoshito Noritome***
}

\begin{abstract}
RESUMO
Introdução: O objetivo deste estudo é avaliar in vitro a transmissão de forças do sistema de retenção do tipo barra-clipe para overdenture, quando duas posições de implantes são simuladas, utilizando-se para isso as técnicas da análise fotoelástica. Métodos: Assim, duas mandíbulas fotoelásticas foram confeccionadas com dois implantes, posicionados na região interforaminal a $22 \mathrm{~mm}$ de distância: modelo 1 - AFIP, modelo fotoelástico com implantes paralelos e orientados verticalmente; e modelo 2 - AFII, modelo fotoelástico com implantes angulados $10^{\circ}$ em relação à linha média da mandíbula. Sobre os implantes foram instalados o sistema de retenção barra-clipe e uma prótese overdenture. Para simulação da mucosa oral, foi interposta, entre a prótese overdenture e a mandíbula fotoelástica, uma camada de $2 \mathrm{~mm}$ de silicone. Cargas de 0,$5 ; 1,0 ; 1,5 ; 2,0$; e 3 bars foram aplicadas e as imagens analisadas. Resultados: Os resultados mostraram que não houve semelhança nas áreas de tensão entre os modelos de resina fotoelástica, quando avaliada a angulação dos implantes. Já o modelo 1 AFIP apresentou maior concentração no ápice dos implantes e o modelo 2 AFII apresentou maior concentração na face mesial dos implantes. Conclusão: Considerando-se as limitações deste trabalho, concluiu-se que o método de análise fotoelástica é de grande valia para a obtenção de informação em relação à biomecânica referente a esse tipo de prótese e sistema de retenção.
\end{abstract}

DESCRITORES: Implantes endoósseos • Implantes dentário endoósseo endodôntico • Mandíbula.

\section{ABSTRACT}

Introduction: The main objective of this study is to appraise in vitro the load transmission in bar-clip retention system for overdenture when 2 implant position are simulated, using for this purpose the photoelastic analysis technique. Methods: Therefore, 2 photoelastic mandibles were manufactured with 2 implants each one, positioned in interforaminal region within $22 \mathrm{~mm}$ of distance: model 1 AFIP, photoelastic model with parallel and vertically oriented implants; and model 2 - AFII, photoelastic model with $10^{\circ}$ angled implants in relation to mandible midline. A bar-clip retention system and an overdenture were fixed over both implants. To simulate oral mucosa were added a $2 \mathrm{~mm}$ silicon layer between overdenture prosthesis and photoelastic mandible. 0.5; $1.0 ; 1.5 ; 2.0$ and 3.0 bars loads were applied and correspondent images were analyzed. Results: Results evidenced that: there is no similarity in loaded areas between photoelastic resin models, when the implant angle is appraised. Since in the model 1 - AFIP, the concentration area of tension was at implant apex and in the model 2 - AFIl was at the distal face. Conclusion: Considering the limitations of this study, it was concluded that photoelastic analysis has a high potential value for obtaining information concerning the biomechanics of this kind of prosthesis and retention system;

DESCRIPTORS: Dental implantation, endosseos • Dental implantation, endosseos, endodontic • Mandible.

\footnotetext{
* Doutoranda do Departamento de Prótese, área de Prótese Parcial Removível, da Faculdade de Odontologia da Universidade de São Paulo - USP.

** Profa. Titular do Departamento de Prótese, da área de Prótese Parcial Removível, da Faculdade de Odontologia da da Universidade de São Paulo - USP.

*** Chefe da divisão para desenvolvimento de produtos do Centro de Tecnologia da Informação Renato Archer.
} 


\section{N T RO DUÇÃ O}

Definida como "uma prótese que obtém retenção de uma ou mais raízes remanescentes sob as selas de próteses totais," as overdentures (sobredentaduras) surgiram como opção de tratamento particularmente nos casos em que existisse a presença de poucos elementos dentários remanescentes (Rutkunas et al. ${ }^{1}$ 2008).

Com o advento das técnicas de osseointegração, implantes têm sido usados do mesmo modo que raízes dentais para reter e suportar overdentures (Naert ${ }^{2}$, 1994). Quando próteses totais convencionais são convertidas em overdentures suportadas por implantes por meio de sistemas de encaixes, um nítido aumento da função mastigatória, retenção, estabilidade, melhora na fonética do paciente e higienização podem ser observados (Ochiai et al. ${ }^{3}$ 2004). Essas vantagens têm significativa importância na explicação do ganho da popularidade do tratamento com overdentures, alternativo às próteses totais convencionais (Rentsch-Kollar et al. ${ }^{4}$ 2010).

Várias são as propostas de tipos de conexão apresentadas pelos fabricantes no sentido de proporcionar resultados positivos quanto ao conforto e função proporcionados pelas overdentures. Dentre os tipos de encaixes, podem ser citados: barra-clipe, tipo bola-copping e magnético. A característica comum entre todos os sistemas consiste em apresentar um componente (posicionado sobre implante ou raiz) e seu correspondente (nylon, plástico, borracha ou metal), que ficam localizados na parte interna da base da prótese, aumentando assim sua estabilidade e retenção, permitindo que os pacientes apresentem padrões de mastigação mais consistentes (Naert et al. ${ }^{5}$ 1999; Walton et al. ${ }^{6}$ 2002).

O planejamento do caso é muito importante para a correta indicação do sistema de encaixe, permitindo que a prótese e os implantes promovam um resultado favorável em longo prazo, sob os aspectos biológicos e funcionais. Os sistemas de retenção podem prover pequenos movimentos verticais e/ou rotacionais da prótese, permitindo que as forças oclusais incidentes sejam dissipadas. Os sistemas de encaixe do tipo barra-clipe e óring seriam os mais comuns e classificados como resilientes (Thayer e Caputo ${ }^{7}$ 1980). O modo de distribuição e dissipação dessas forças dependeria basicamente do sistema utilizado, número de implantes e suas inclinações. Uma condição ideal seria a incidência da força uniformemente tanto sobre os sistemas de retenção quanto sobre os implantes, mucosa e rebordo alveolar. Isso evitaria a sobrecarga em qualquer parte desse conjunto (Federick et al. ${ }^{8}$ 1996; Ochiai et al. ${ }^{3}$ 2004). Dessa forma os implantes tendem a transmitir e distribuir maior quantidade de carga para o osso adjacente (Kenney et al. ${ }^{9}$ 1998). Se a força incidente exceder o limite fisiológico, pode resultar em sobrecarga com consequente perda do implante, já que esse não está preparado para suportar forças excessivas (Ochiai et al. ${ }^{3}$ 2004). Portanto, torna-se essencial que as questões referentes ao comportamento biomecânico dos implantes e como reagem os tecidos circunvizinhos quando submetidos às cargas sejam investigadas, pois estão diretamente relacionadas à preservação dos tecidos de suporte.

Existem algumas metodologias de estudo para avaliar a incidência dessas forças, tais como: modelos teóricos de elementos finitos e modelos experimentais como strain gauge e a técnica da análise fotoelástica. A carência de dados na literatura, além dos fatores acima expostos, levou a desenvolver este trabalho de pesquisa com o propósito de estudar a transmissão de forças quando o sistema de retenção do tipo barra-clipe para overdentures retidas por implantes é utilizado, simulando duas posições de implantes e utilizando para isso a metodologia de análise fotoelástica, visto que a sobrecarga pode criar condições desfavoráveis, diminuindo a longevidade dos implantes e consequentemente da prótese, com insucesso nas reabilitações bucais com overdentures.

\section{MÉTODOS}

Foi utilizado neste trabalho o sistema de encaixe do tipo barra-clipe, empregado como sistema de retenção de próteses tipo overdentures. No Quadro 1, estão relacionados o fabricante, o sistema de encaixe e
PIGOZZO MN,

LAGANÁ DC,

NORITOME PY.

AVALIAÇÃO DAS

TENSÕES Às

ESTRUTURAS DE

SUPORTE, POR

MEIO DO MÉTODO

FOTOELÁSTICO,

DECORRENTES

DAS FORÇAS

APLICADAS SOBRE

OVERDENTURES

RETIDAS EM

IMPLANTES COM

SISTEMA DE

ENCAIXE BARRA -

CLIPE

123

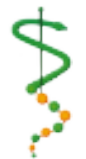

REVISTA DE ODONTOLOGIA DA UN I VERS I DADE CIDADE DE SÃO PAULO

$2010 ; 22(2): 122-$

33, MAI-AGO 
PIGOZZO MN,

LAGANÁ DC,

NORITOME PY.

AVALIAÇÃO DAS

TENSÕES ÀS

ESTRUTURAS DE

SUPORTE, POR

MEIO DO MÉTODO

FOTOELÁSTICO,

DECORRENTES

DAS FORÇAS

APLICADAS SOBRE

OVERDENTURES

RETIDAS EM :

IMPLANTES COM

SISTEMA DE

ENCAIXE BARRA-

CLIPE

$124 \ldots$

REVISTA DE

ODONTOLOGIA DA

UNIVERSIDADE

CIDADE DE SÃO

PAULO

2010; 22(2): 122-

33, MAI-AGO o número de amostras.

Para a realização desta pesquisa, foi utilizado o crânio fotoelástico elaborado em 2004 (Fig.1), a partir do qual foi produzida a mandíbula, para a confecção dos corpos de prova e posterior realização dos ensaios concernentes a esta pesquisa.

Para realização desta investigação, foram retirados os dentes da mandíbula do crânio fotoelástico para que os alvéolos pudessem ser preenchidos com silicone laboratorial Zetalabor (Zhermack Clínical, Badia Polesine, Rovigo, Itália), permitindo-se, assim, que fosse obtida uma mandíbula com as características de um paciente desdentado. A mandíbula do crânio fotoelástico, com os alvéolos preenchidos com silicone, foi colocada no interior de um recipiente plástico. Assim, a base da mandíbula foi fixada com cola, à base de cianoacrilato (Superbonder, Henkel Co, Düsseldorf, Alemanha), na tampa desse recipiente e vazado em seu entorno silicone industrial, manipulado de acordo com as orientações do fabricante.

Após 48 horas, o modelo foi retirado do molde, sob o qual foi vertida resina epóxi com adição de carbonato de cálcio na proporção de 100:30 (Epoxiglass, São Paulo, SP, Brasil), para a obtenção de uma réplica da mandíbula do crânio fotoelástico. Para simular a reabsorção das tábuas ósseas desses alvéolos após a exodontia, foi realizada a redução da mandíbula, com a ponta maxicut e taça para acabamento e polimento, simulando-se, assim, uma perda óssea característica de pacientes totalmente desdentados.

$\mathrm{Na}$ mandíbula reproduzida em resina epóxi, delimitou-se a área basal para a confecção da base de prova que permitiu a obtenção do registro intermaxilar. $\mathrm{O}$ isolamento do modelo foi realizado com Cel-Lac (SS White Artigos Dentários, Rio de Janeiro, RJ, Brasil). A base de prova foi confeccionada com resina acrílica ativada quimicamente (Artigos Odontológicos Clássico Ltda, Campo Limpo Paulista, SP, Brasil) e o plano de orientação em cera 7 (Epoxiglass Ind. e Com. de Produtos Químicos Ltda, Diadema, SP, Brasil) seguindo o alinhamento dos dentes superiores do crânio fotoelástico. A dimensão vertical de oclusão foi obtida posicionando-se o côn- dilo da mandíbula dentada na fossa mandibular do crânio fotoelástico, e essa altura do terço inferior do crânio foi mensurada com o compasso de Willis. Em seguida, o côndilo da mandíbula desdentada, com a base de prova e o plano de orientação em cera, foi posicionado na fossa mandibular do crânio fotoelástico. O conjunto-base de prova e plano de orientação em cera foi ajustado na altura mensurada anteriormente, permitindo, desse modo, o registro da oclusão pela maxila dentada original do crânio em questão.

O arco dentário superior do crânio fotoelástico foi reproduzido com silicone de adição Flexitime (Heraus Kulzer $\mathrm{GmbH}$, Hanau, Alemanha), utilizando-se a técnica de transferência direta, por meio da confecção de uma moldeira individual em cera 7 e cera utilidade, que otimizou a montagem em articulador do modelo superior. Assim, os modelos, superior e inferior, foram montados em articulador semiajustável, previamente ajustado: ângulo de Bennett em 15\% ângulo da guia condilar em $30^{\circ}$, plataforma incisal em $0^{\circ}$ e distância intercondilar pequena.

Após a montagem dos modelos no articulador, foi realizada a montagem dos dentes artificiais de estoque, marca Orthosit, modelo A9 (anteriores) e N6 (posteriores), que permitiu a obtenção de uma prótese total e da guia cirúrgica.

A guia cirúrgica estabeleceu o eixo de inserção da prótese, além de permitir estabelecer o correto posicionamento dos implantes no futuro modelo fotoelástico. Assim, essa guia foi posicionada sobre a mandíbula de resina epóxi para que fossem realizadas as perfurações na região dos caninos, utilizando-se para tanto uma Fresadora Microtech para permitir o paralelismo entre eles. As marcações para instalação dos análogos dos implantes foram realizadas entre foramens mentuais, ficando $3 \mathrm{~mm}$ aquém dos limites anatômicos destes, a aproximadamente uma distância de $22 \mathrm{~mm}$ um em relação ao outro, a partir de seus centros (Sadowsky ${ }^{10}$ 2000).

Foram realizadas perfurações para permitir a confecção de dois modelos da mandíbula em resina fotoelástica: um modelo com implantes posicionados na vertical, paralelos entre si (Modelo 1), e outro em 
que os implantes apresentavam divergência de $10^{\circ}$ em relação à linha média (Modelo 2). Para a confecção do Modelo 2, foi utilizado um transferidor para assegurar a divergência de $10^{\circ}$ entre os implantes (Federick e Caputo ${ }^{8}$ 1996).

Assim, sobre os análogos instalados na mandíbula de resina epóxi foram parafusados os transferentes, também da marca Conexão, e unidos com fio dental formando uma malha que, em seguida, foi solidificada com resina acrílica duralay ativada quimicamente. O conjunto unido com duralay (Duralay Resiliance - MFG Co, Chicago, EUA) foi então seccionado na região interimplantes com disco de aço, para novamente serem unidos. Dessa forma, procurou-se minimizar possíveis distorções decorrentes da contração de polimerização da resina.

No interior de um recipiente plástico, posicionou-se o modelo da mandíbula em resina epóxi com os análogos de implantes e os seus respectivos transferentes parafusados, de forma que a base da mandíbula fosse fixada na tampa do recipiente com adesivo à base de cianoacrilato. Em seguida, o recipiente foi recortado na altura dos côndilos e canudos de plástico foram acoplados nas pontas dos parafusos dos transferentes, para que ultrapassassem a altura da abertura feita no recipiente plástico, permitindo o acesso aos parafusos mesmo após a polimerização do silicone.

Para a realização da moldagem foi vertido, no interior do recipiente plástico em torno da mandíbula, silicone Industrial Aerojet (Aerojet Ind. de Plásticos, São Paulo, SP), manipulado de acordo com as recomendações do fabricante. Após a polimerização do silicone, os parafusos dos transferentes foram soltos, a tampa do recipiente plástico removida e o modelo da mandíbula em resina foi retirado.

Os dois implantes foram parafusados aos transferentes com uma chave para implante no formato hexagonal. Os implantes de titânio selecionados foram os do tipo Master Screw, na forma rosqueável, com $13 \mathrm{~mm}$ de comprimento, 3,75mm de diâmetro no seu corpo, 4,1 mm de diâmetro na sua plataforma e encaixe na forma de hexágono externo com $0,7 \mathrm{~mm}$ de altura e $2,7 \mathrm{~mm}$ de distância entre os lados do hexágono; a partir desse molde obteve-se o modelo da mandíbula em resina fotoelástica.

Assim, para a manipulação da resina fotoelástica PL-2, foram seguidas as instruções do fabricante (Vishay Measurements Group, Raleigh, NC, EUA), adicionandose cuidadosamente os dois componentes, na proporção de 50:50, 150g de resina PL-2 base e $150 \mathrm{~g}$ de catalisador, proporcionados em dois recipientes e pesados em uma balança digital. Os componentes foram misturados com um bastão de vidro, com movimentos circulares de baixo para cima, com o cuidado de não introduzir maior quantidade de bolhas de ar, até se obter uma mistura homogênea. Após essa fase, colocou-se a mistura na bomba a vácuo para eliminar as bolhas de ar que foram incluídas durante a espatulação. A resina foi lentamente vertida no molde de silicone e, em seguida, levados para a câmara a vácuo por alguns minutos, a fim de eliminar as possíveis bolhas que pudessem ter sido incluídas. Aguardou-se 72 horas, em temperatura ambiente, para a completa polimerização da resina fotoelástica e, em seguida, realizou-se a desinclusão do modelo. Após esse período, os parafusos fixadores dos transferentes foram soltos e o modelo fotoelástico foi removido do molde.

Para o enceramento das barras, foram utilizados: dois pilares cilíndricos do tipo "UCLA" calcinável sem hexágono externo (sistema antirrotacional), com diâmetro de $3,75 \mathrm{~mm}$; discos de carborundum para reduzir suas alturas em $4 \mathrm{~mm}$, dois parafusos de titânio para UCLAs, e chave hexagonal com diâmetro de 1,17. Após o enceramento das duas barras, foram realizados os processos de inclusão, fundição, acabamento e polimento. Todas as etapas foram realizadas por um técnico de laboratório (Laboratório Smiles Factory São Paulo, SP). A acrilização das próteses foi realizada de forma convencional.

Após a finalização da prótese overdenture, cada barra foi instalada na sua respectiva mandíbula de acordo com a posição dos implantes, ou seja, a barra encerada sobre a mandíbula que apresentava implantes paralelos, após a fundição, foi posicionada nessa mesma mandíbula e
PIGOZZOMN,

LAGANÁ DC,

NORITOME PY.

AVALIAÇÃO DAS

tensões às

ESTRUTURAS DE

SUPORTE, POR

MEIO dO MÉTODO

FOTOELÁSTICO,

DECORRENTES

DAS FORÇAS

APLICADAS SOBRE

OVERDENTURES

RETIDAS EM

IMPLANTES COM

SISTEMA DE

ENCAIXE BARRA -

CLIPE
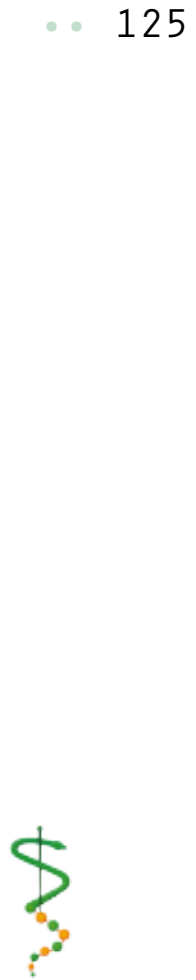

ReVISTA DE

ODONTOLOGIA DA

Universidade

CIDAde de São

PAuLO

2010; 22(2): $122-$

33, MAI-AGO 
PIGOZZOMN,

LAGANÁ DC,

NORITOME PY.

AVALIAÇÃO DAS

TENSÕES ÀS

ESTRUTURAS DE

SUPORTE, POR

MEIO DO MÉTODO

FOTOELÁSTICO,

DECORRENTES

DAS FORÇAS

APLICADAS SOBRE

OVERDENTURES

RETIDAS EM

IMPLANTES COM

SISTEMA DE

ENCAIXE BARRA-

CLIPE

\section{6}

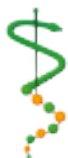

REVISTA DE

ODONTOLOGIA DA

UNI VERS I DADE

CIDADE DE SÃO

PAULO

$2010 ; 22(2): 122-$

33, MAI-AGO o mesmo foi feito para a mandíbula de resina fotoelástica de implantes inclinados. Após a instalação das barras, os parafusos UCLAs receberam um torque de $32 \mathrm{~N}$, valor este recomendado pelo fabricante.

A captura do clipe no interior da loja foi realizada com resina acrílica autopolimerizável, permitindo, assim, que a prótese fosse finalmente e instalada sobre a barra. Para permitir uma melhor visualização das franjas fotoelásticas durante os ensaios, foi recortada, com uma broca maxicut, a porção anterior da prótese, correspondente à região dos implantes.

Em seguida, uma camada de silicone de aproximadamente $2 \mathrm{~mm}$ foi colocada na região posterior e interior da sela protética fotoelástica, com a finalidade de simular a resiliência da mucosa bucal (Thayer e Caputo $^{11}$ 1979, Thayer e Caputo ${ }^{7}$ 1980, Federick e Caputo ${ }^{8}$ 1996, Kenney e Richards $^{9}$ 1998, Sadowsky e Caputo ${ }^{10} 2000$, Ochiai et al. ${ }^{3}$ 2004). Esse procedimento foi realizado, baseado no fato de que materiais de moldagem à base de silicone apresentam módulo de elasticidade de aproximadamente $2 \mathrm{MPa}$, semelhante ao apresentado por tecidos moles tais como a pele, equivalente a aproximadamente 1 MPa (Porter et al. ${ }^{12}$ 2002).

A mandíbula foi adaptada e fixada, com resina acrílica autopolimerizável, na base de acrílico que compõe o crânio fotoelástico e, finalmente, antes da realização dos ensaios fotoelásticos, os ajustes oclusais foram realizados utilizando-se folhas de carbono Accufilm II. Esse ajuste oclusal teve a finalidade de evitar a concentração de cargas, devido a contatos prematuros que pudessem ocorrer durante os ensaios fotoelásticos.

De acordo com o descrito, para a realização dos ensaios fotoelásticos, foram construídos dois modelos: um constituído por uma mandíbula contendo dois implantes paralelos entre si, sobre os quais ancorou-se uma prótese overdenture por meio do sistema de encaixe de retenção barra-clipe - Modelo 1; e o outro foi constituído por uma mandíbula contendo dois implantes inclinados, divergentes $10^{\circ}$ em relação à linha média, sobre os quais ancorou-se a mesma prótese overdenture por meio do sistema de encaixe de reten- ção do tipo barra-clipe - Modelo 2. Essas situações foram preparadas para serem avaliadas com o auxílio do método fotoelástico, visualizadas e fotografadas em vista frontal, independente da sobreposição de imagens que ocorreram nessa situação, uma vez que todas as comparações e associações foram feitas nessa mesma posição e provavelmente com as mesmas sobreposições.

Assim, os modelos fotoelásticos foram mergulhados no recipiente contendo o óleo mineral, interposto entre o polarímetro e o filtro polarizador e, com a fonte de luz incidente, foram realizadas as tomadas fotográficas: (I) inicial com incidência 0 de carga; (II) com aplicação de 0,5 bar; (III) 1 bar; (IV) 1,5 bar; (V) 2 bares e (VI) 3 bares, respectivamente para cada mandíbula.

A seguir, os modelos fotoelásticos foram nomeados de acordo com a inclinação dos implantes, seguidos da carga aplicada: Modelo 1 - AFIP, para descrição do modelo de análise fotoelástica que apresenta implantes paralelos (AFIP0; AFIP0,5; AFIP1; AFIP1,5; AFIP2; AFIP3); e Modelo 2 - AFII, para o modelo de análise fotoelástica que apresenta implantes inclinados (AFIIO; FIIO,5; AFII1; AFII1,5; AFII2; AFII3).

\section{RESULTADOS}

Os resultados foram obtidos pela avaliação das tensões provenientes da aplicação das cargas sobre os corpos de prova, compostos pelos modelos fotoelásticos e overdentures, retidas por sistemas de encaixe tipo barra-clipe, associadas a implantes e suportadas por estes e rebordo residual, em posição de máxima interscupidação, em todas as condições de carregamento.

Para análise dos resultados foi realizado o registro fotográfico do modelo fotoelástico anterior à aplicação de cargas de uma vista frontal, independente da sobreposição de imagens que ocorreram nessa situação, uma vez que todas as comparações e associações foram feitas, nessa mesma posição e, provavelmente, com as mesmas sobreposições.

A análise dos resultados foi realizada tendo-se como ponto de partida as imagens iniciais da mandíbula: com implan- 


\begin{tabular}{cccc} 
Fabricante/Marca & Sistema/Encaixe & Posição dos implantes & № de Amostras \\
Conexão ${ }^{\circledR}$ Sistemas de Prótese & Tipo Barra-clipe & Paralelos & 1 \\
Conexão $0^{\circledR}$ Sistemas de Prótese & Tipo Barra-clipe & Divergentes $10^{\circ}$ & 1 \\
\hline
\end{tabular}

Quadro 1 - Marca comercial/fabricante, sistema de encaixe, posição dos implantes e número de amostras utilizadas no trabalho

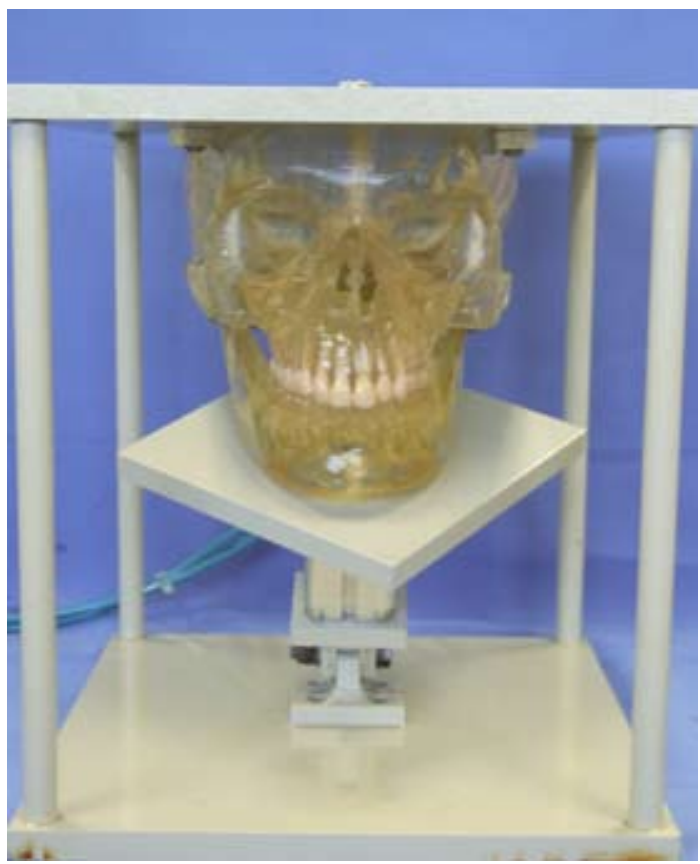

Figura 1 - Crânio fotoelástico

tes paralelos e ausência de aplicação de carga e, em seguida, com os implantes inclinados e ausência de aplicação de carga, respectivamente AFIP0 e AFIIO. As regiões escolhidas para análise e comparação foram: região inter-forame mentoniano e rebordo desdentado posterior.

Todas as imagens referentes ao Modelo 1 foram agrupadas na Figura 2.

Assim, nota-se que o corpo da mandíbula do AFIP0, sem aplicação de carga, apresenta imagens coloridas em algumas regiões, delimitadas na Figura 2. Essas áreas coloridas, que sugerem áreas de tensão são o resultado da manipulação e homogeneização dos componentes da resina fotoelástica; da confecção do corpo de prova e ajuste da prótese sobre o modelo, como também da fixação deste à base de acrílico, suporte do modelo na máquina de aplicação de cargas e da manipulação da mandíbula durante os ajustes oclusais com a maxila do crânio. Essas tensões são passíveis de ocorrer, mesmo que todos os cuidados sejam tomados para evitar o surgimento das franjas, anterior a realização dos ensaios fotoelásticos. Assim, a sequência adotada para análise dos resultados foi planejada para avaliar a intensidade das tensões geradas durante a aplicação das cargas, levando-se sempre em consideração as tensões inicialmente presentes nos modelos.

O Modelo 1, Figura 2, AFIP0 - sem aplicação de carga, apresentou colorações fotoelásticas no ápice do implante posicionado à direita na figura. $\mathrm{O}$ implante posicionado à esquerda apresenta-as ao longo do seu corpo, mais esparsas na sua mesial e mais delimitadas na distal. A coloração ao longo do rebordo foi maior no lado esquerdo, sendo que o lado direito não as apresentou. $\mathrm{O}$ carregamento de 0,5 bar - AFIP0,5, Figura 2, não promoveu uma alteração significativa no padrão de distribuição de tensões quando comparado com a imagem do AFIP0. Porém, o implante posicionado à esquerda da figura apresentou um aumento na intensidade e uma maior delimitação das franjas. Durante o carregamento de 1,0 bar - AFIP1, Figura 2, houve um aumento na intensidade das tensões nas áreas anteriormente analisadas, proporcionais à carga aplicada. Porém, ocorreu uma alteração nos padrões de distribuição de tensão na região posterior do rebordo, com o aparecimento de áreas de tensão, como indicado pelas setas.

O registro fotográfico mostra que, quando houve aplicação de 1,5 bar AFIP1,5, (Figura 2), ocorreu um aumento na intensidade das tensões proporcional à carga aplicada, bem como a formação de franjas com formato definido na região posterior do rebordo, como indicado pelas setas. Porém, não ocorreu alteração no padrão de distribuição dessas tensões, que permaneceram locadas nas mesmas regiões do AFIP1. O ensaio registrado para a aplicação de carga de 2 bar - AFIP2, (Figura 2), mostra que ocorreu um aumento na intensidade das tensões, proporcional
PIGOZZOMN,

LAGANÁ DC,

NORITOME PY.

AVALIAÇÃO DAS

TENSÕES ÀS

ESTRUTURAS DE

SUPORTE, POR

MEIO DO MÉTODO

FOTOELÁSTICO,

DECORRENTES

DAS FORÇAS

APLICADAS SOBRE

OVERDENTURES

RETIDAS EM

IMPLANTES COM

SISTEMA DE

ENCAIXE BARRA-

CLIPE

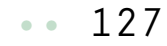

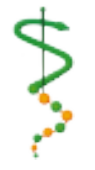

REVISTA DE ODONTOLOGIA DA UN I VERS I DADE Cidade de São PAULO

$2010 ; 22(2): 122-$

33, MAI-AGO 


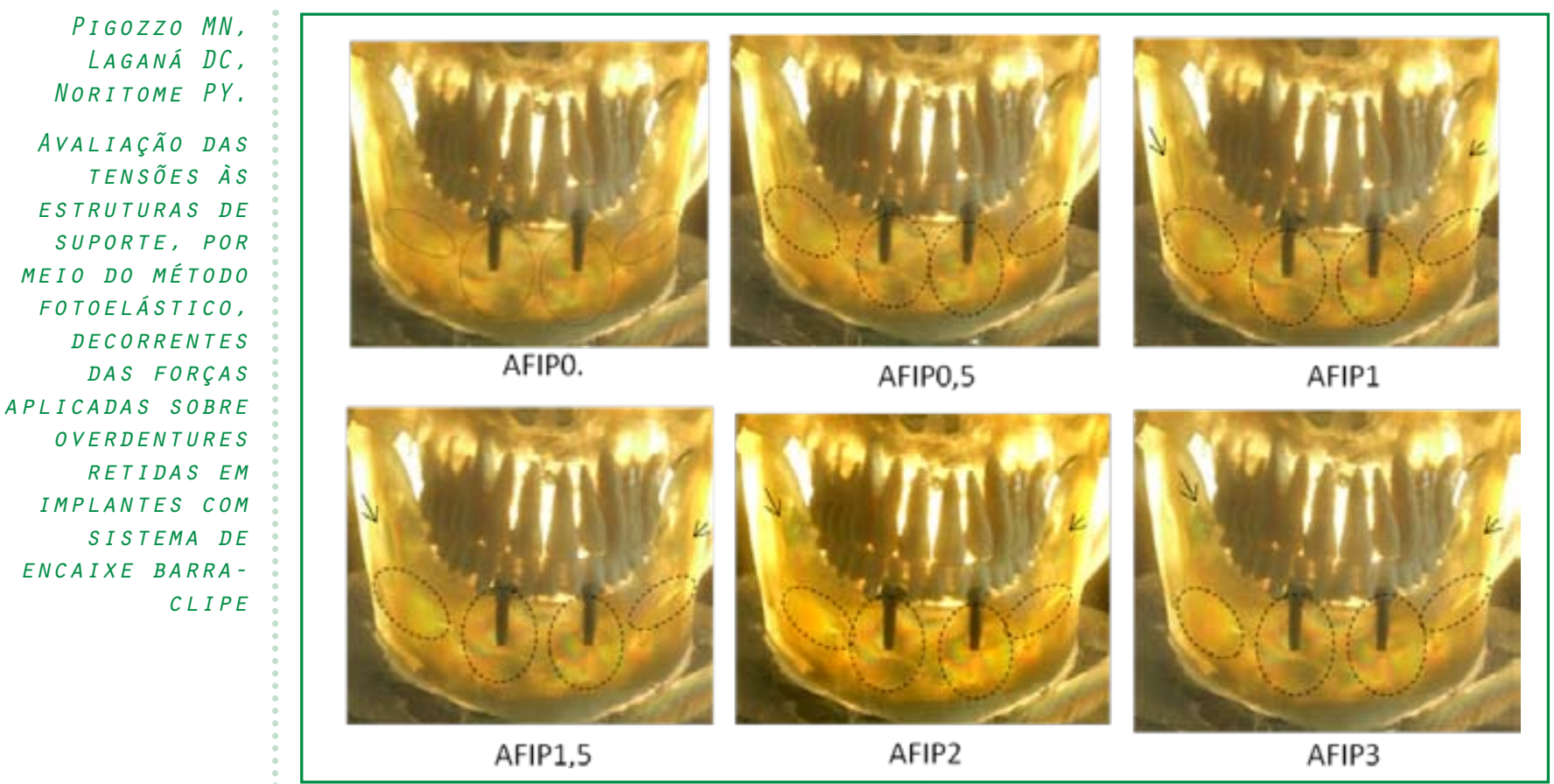

Figura 2 - AFIP: Overdenture retida por barra-clipe sobre dois implantes paralelos, seguido da carga aplicada

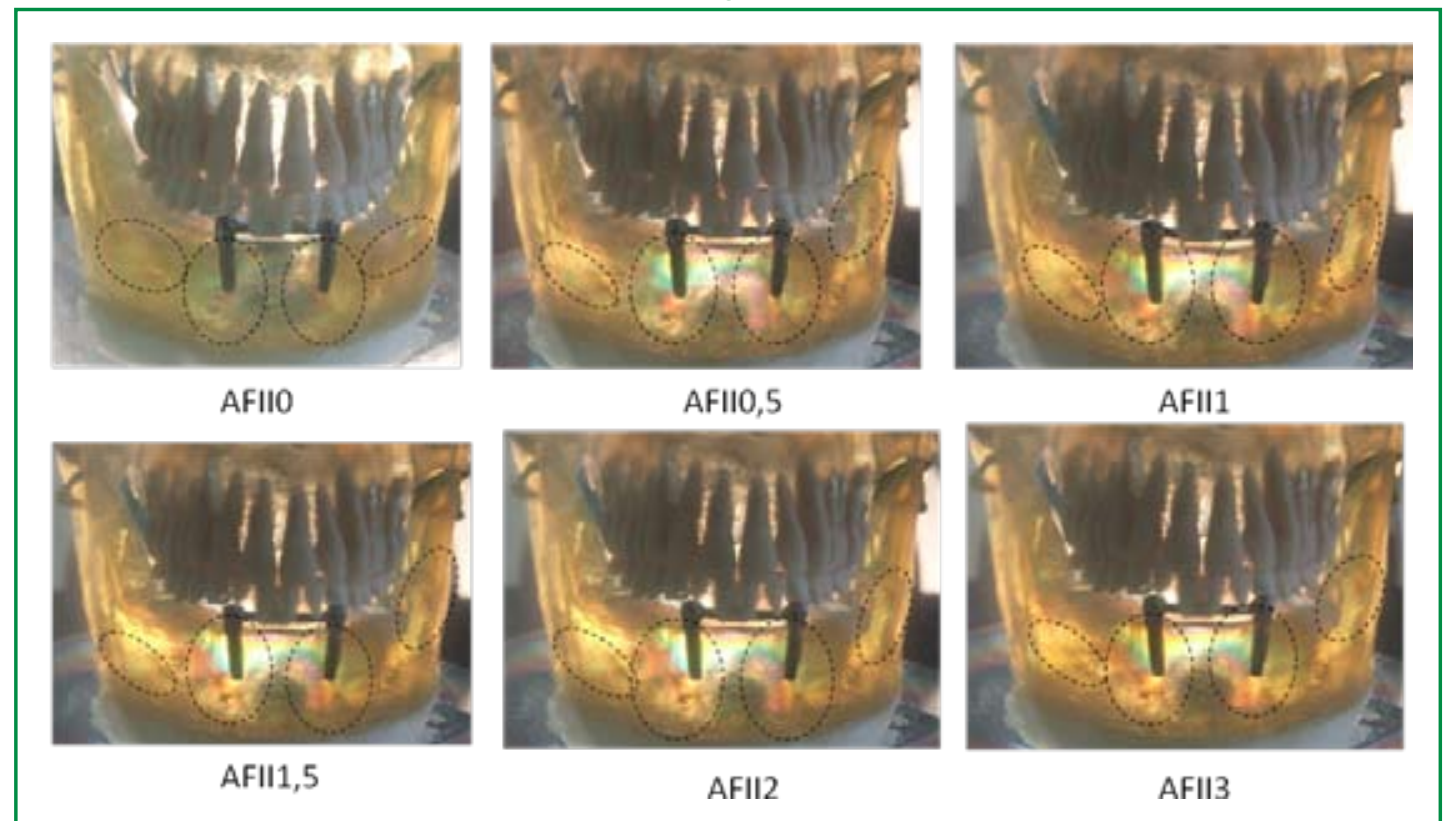

Figura 3 - AFII: Overdenture retida por barra-clipe sobre dois implantes angulados, seguido da carga aplicada

à carga aplicada, Na Figura 2, com carregamento de 3 bar - AFIP3, percebe-se que as tensões se intensificaram na região do trígono retromolar. Com esse carregamento ocorreu fratura da resina da overdenture na região de canino (elemento 33), logo acima do implante posicionado à direita.

Para o Modelo 2 - AFII, as regiões escolhidas para análise e comparação foram as mesmas realizadas para o Modelo 1.

A avaliação do corpo de prova, Mo- delo 2, com implantes inclinados $10^{\circ} \mathrm{em}$ relação à linha média e ausência de carregamento - AFIIO, foi realizada levando-se em consideração as mesmas observações feitas em relação às tensões residuais do modelo, justificadas para o corpo de prova AFIP0. Na Figura 3, AFIIO, o comportamento apresentado perante a ausência de aplicação de carga demonstrou uma concentração moderada de tensão no implante posicionado à esquerda na figura. 
Já no implante posicionado à direita na figura, pôde-se observar uma baixa distribuição de tensão localizada no seu ápice. Não houve a formação de franjas consistentes ao longo do rebordo alveolar, tanto do lado esquerdo quanto do direito. $\mathrm{Na}$ Figura 3, com aplicação de carga de 0,5 bar - AFII0,5, observou-se aumento na intensidade das tensões, seguido de alteração no padrão de distribuição, quando comparado com a AFIIO. O implante posicionado à esquerda da figura apresentou um aumento na intensidade da tensão, de acordo com a lógica de que, aumentandose a intensidade da incidência da carga, aumenta-se a concentração de tensão no modelo. O implante posicionado à direita da figura apresentou um aumento na concentração de tensão na sua mesial, fazendo com que as franjas localizadas na mesial de cada implante se encontrassem na região mentoniana. Observam-se, ainda, áreas de tensão na região do trígono retromolar, no lado direito da figura, e na região de rebordo alveolar, no lado esquerdo, tais como circunscritos na figura. Já carregamento de 1,0 bar - AFII1,0, visto na Figura 3, manteve o padrão de distribuição de tensão, tanto na região dos implantes como no rebordo residual; porém ocorreu aumento na intensidade das tensões, que se detecta pela maior luminosidade das áreas, principalmente na dos implantes, quando comparado com a II0,5. Com a aplicação de 1,5 bar de carga - AFII1,5, as tensões se intensificaram, como se percebe na Figura 3. Tensões foram geradas na distal do implante posicionado à direita na figura. Não houve alteração significativa nos padrões de distribuição das tensões, quando comparado com a AFII1,0. O mesmo ocorreu com a aplicação de carga de 2 bares - AFII2, em que se pode observar comportamentos semelhantes entre os carregamentos de 1,5; 2,0; e 3 bars.

\section{I SCUSSÃO}

O corpo de prova AFIP0 da Figura 2 apresentou franjas coloridas, mais concentradas na região dos implantes e na região do rebordo alveolar. Sendo assim, as avaliações do comportamento das tensões foram feitas a partir dessa imagem inicial.
Na observação do Modelo 1 - AFIP, quando submetido às cargas de 0 a 3 bars, observou-se uma concentração de tensão nos ápices dos implantes e região de rebordo alveolar residual. A presença de tensões no ápice dos implantes é explicada pelo fato de que, quando submetidos à carga, os implantes têm uma tendência natural à intrusão. Essa tentativa de penetração do implante na resina fotoelástica é impedida pela resistência da resina, a sua dureza. Assim, são geradas as áreas de tensão no ápice e, portanto, as franjas fotoelásticas (Thayer e Caputo ${ }^{11} 1979$ Thayer e Caputo ${ }^{7}$ 1980, Gonini ${ }^{13}$ 2002).

Já as tensões na região de rebordo alveolar ocorrem ao longo de toda a delimitação da área basal da prótese. Esse comportamento reforça que os conceitos básicos e científicos relacionados à construção das próteses totais convencionais devem ser preservados e aplicados nessa modalidade de prótese sobre implantes (Gomes ${ }^{14}$ em 2005). Os princípios relacionados à delimitação da área basal são de extrema importância para a preservação do tecido ósseo remanescente, principalmente a extensão da base protética, que, quando situada nos limites preconizados, proporciona melhor distribuição de carga. Dessa forma, cada unidade de área recebe menor intensidade de carga, preservando o rebordo quanto ao processo de reabsorção óssea.

De acordo com Kenney e Richards ${ }^{9}$ (1998), há uma principal diferença no padrão de distribuição de tensão ao longo do corpo do implante quando a carga é aplicada axialmente, em todo o arco dentário, isso porque a concentração de tensão nos implantes não é diminuída; pelo contrário, há uma concentração de tensão em seu ápice. Isso não ocorre quando a carga é aplicada pontualmente na região posterior, principalmente em primeiros molares, quando o sistema de retenção barra-clipe tende a rotacionar e a tensão tende a ser transferida perpendicularmente para a região posterior do rebordo (Porter et al. ${ }^{12}$ 2002). Da mesma forma, Federick e Caputo ${ }^{8}$ em 1996, avaliaram a distribuição de tensão em overdentures retidas por diferentes sistemas de retenção, aplicando a carga sobre os implantes, no segundo
PIGOZZO MN,

LAGANÁ DC,

NORITOME PY.

AVALIAÇÃO DAS

tensões às

ESTRUTURAS DE

SUPORTE, POR

MEIO dO MÉTODO

FOTOELÁSTICO,

DECORRENTES

DAS FORÇAS

APLICADAS SOBRE

OVERDENTURES

RETIDAS EM

IMPLANTES COM

SISTEMA DE

ENCAIXE BARRA-

CLIPE
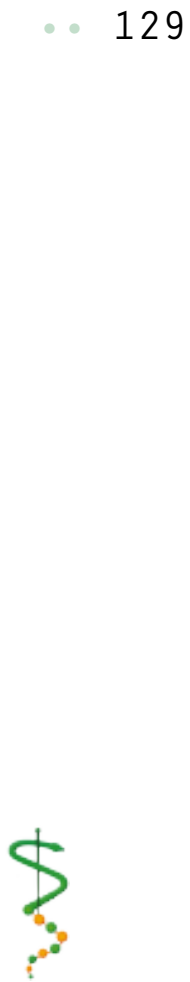

ReVISTA DE

ODONTOLOGIA DA

UNIVERS I DADE

CIDAde de São

PAULO

2010；22(2)：122-

33, MAI-AGO 
PIGOZZO MN,

LAGANÁ DC,

NORITOME PY.

AVALIAÇÃO DAS

TENSÕES ÀS

ESTRUTURAS DE

SUPORTE, POR

MEIO DO MÉTODO

FOTOELÁSTICO,

DECORRENTES

DAS FORÇAS

APLICADAS SOBRE

OVERDENTURES

RETIDAS EM

IMPLANTES COM

SISTEMA DE

ENCAIXE BARRA-

$C L I P E$

130

REVISTA DE

ODONTOLOGIA DA

UNIVERSIDADE

CIDADE DE SÃO

PAULO

$2010 ; 22(2): 122-$

33, MAI-AGO pré-molar e no segundo molar. Os autores concluíram que, quanto mais posterior a aplicação da carga, maior a concentração de tensão no rebordo alveolar posterior e menor a concentração de tensão na região anterior dos implantes. Assim, alguns relatos são encontrados na literatura de autores que observaram maior concentração de tensão no rebordo alveolar residual posterior, em detrimento de uma menor concentração de tensões na região anterior dos implantes (Ichikawa et al. ${ }^{15}$ 1996; Covaciuc $^{16}$ 2002; Sadowsky e Caputo ${ }^{10}$ 2004), situação essa que não foi evidenciada neste estudo.

Dessa forma, a diferença na concentração de tensão, quando comparadas as regiões de rebordo alveolar posterior e a região anterior de implante, pode ser explicada pelo fato de a aplicação da carga ter ocorrido axialmente em todo o arco dentário. Luo et al. ${ }^{17}$ em 1998 e Kenney e Richars $^{9}$ em 1998 afirmaram que a maior concentração de tensão na região dos implantes se deve ao fato de estarem unidos por meio do sistema de retenção barra-clipe, que transmite uma maior concentração de tensão na região anterior quando comparado com o rebordo alveolar residual posterior. Bortloli J ${ }^{18}$ em 2004, comparou a transmissão de tensão em rebordo alveolar em overdentures retidas por sistema de retenção barra-clipe e óring. Assim, o sistema de retenção barra-clipe foi responsável por gerar uma maior concentração de tensão na região anterior dos implantes (Piagge $^{19}$ 2002; Silva et al. ${ }^{20}$ 2010). Resultados semelhantes ao deste trabalho foram relatados por Thayer e Caputo ${ }^{11}$ em 1977, que descreveram distribuição de tensão ao longo de todo o rebordo e uma maior concentração de tensão nos ápices de remanescentes radiculares quando uma overdenture é retida pelo sistema barra de Dolder.

Porém, a literatura relata, também, que a menor concentração de tensão no rebordo alveolar residual posterior pode ter ocorrido devido à interposição da camada de silicone, cujo principal objetivo é simular a mucosa oral e isso ocorre devido a sua resiliência e capacidade de absorver tensões (Thayer e Caputo ${ }^{7}$ 1980; Ichikawa et al. ${ }^{15} 1996$; Sadowsky e Caputo ${ }^{10}$ 2000).
Já o corpo de prova AFIIO, da Figura 3, apresentou franjas coloridas, mais concentradas na região dos implantes, sem a formação de franjas consistentes ao longo do rebordo alveolar residual posterior, tanto do lado esquerdo quanto do direito. Sendo assim, as avaliações do comportamento das tensões foram feitas a partir dessa imagem inicial.

Na observação do Modelo 2 - AFIl, quando submetido às cargas de 0 a 3 bars, observou-se um aumento na concentração de tensão na região anterior, nos implantes, e uma baixa concentração de tensão no rebordo alveolar residual posterior. Thayer e Caputo ${ }^{11}$ (1979) afirmaram que, após a aplicação da carga, os implantes tendem a intruir na resina fotoelástica, e são impedidos pela resistência desta, provocando a formação de franjas fotoelásticas nos ápices dos implantes, devido à maior concentração de tensões. Quando o implante apresenta uma inclinação e recebe uma força perpendicular ao plano oclusal, ocorre a mesma tendência à intrusão. Porém, devido à angulação do implante, este tende a descer inclinado, ocorrendo, assim, a formação de franjas, não só no ápice mas também ao longo do seu corpo na face distal; isso porque o implante não tende a intruir apenas axialmente, paralelo ao carregamento (Federick e Caputo ${ }^{8}$ 1996). Além disso, Menicucci et al. ${ }^{21}$ em 1998, afirmaram que ocorre uma concentração de tensão na região anterior de mandíbula, quando overdentures são ancoradas por meio de sistema de retenção unido, tais como barra-clipe, devido à deformação da mandíbula durante a aplicação da carga, que gera uma torção na parte central, onde estão ancorados os implantes, o que pode explicar essa alta concentração de tensão gerada nos implantes.

Além disso, Celik e Vludag22 em 2007, afirmaram que as posições dos ápices dos implantes, após a inclinação dos mesmos ficam mais próximas, provocando a soma ou encontro das tensões nessa região, o que explica a maior concentração de tensão, também, quando comparado com o modelo fotoelástico que apresenta implantes paralelos, uma vez que nesse modelo a concentração de tensão na região 
anterior dos implantes foi menor quando comparado com o Modelo 2.

A pouca concentração de tensão no rebordo alveolar pode ter ocorrido, como explicitado anteriormente, pela presença do silicone, simulando mucosa oral (Thayer e Caputo ${ }^{7}$ 1980; Ichikawa et al. ${ }^{15} 1996$; Sadowsky e Caputo ${ }^{10}$ 2000) ou pelo fato do sistema de retenção barra-clipe gerar maior concentração de tensão na região dos implantes (Kenney e Richards ${ }^{9}$ 1998, Piagge $^{19}$ 2002; Silva et al. ${ }^{20} 2010$; Thayer e Caputo ${ }^{11}$ 1979).

Modelos experimentais de tensão, tais como os de análise fotoelástica, são amplamente utilizados em Odontologia para avaliação de tensões, porém o método apresenta suas limitações. Assim, a resina utilizada para simulação da estrutura óssea apresenta diferenças em relação à isotropia e homogeneidade quando comparada a um osso real, assumindo que todas as estruturas são homogêneas (Eser et al. ${ }^{23}$ 2009). Além disso, é considerado um contato perfeito entre implante e estrutura óssea, como se a osseointegração ocorresse da mesma forma, e 100\%, ao longo de todo o corpo do implante (Menicucci et al. ${ }^{21}$ 1998); também é desconsiderado o fato de que a osseointegração é, na realidade, um processo dinâmico (Ochiai et al. ${ }^{3}$ 2004). Além disso, a análise tridimensional da análise fotoelástica é feita através de imagens, que apresentam apenas duas dimensões, resultando, assim, em outra limitação (Kenney e Richards ${ }^{9}$ 1998).

As cargas simuladas foram aplicadas axialmente (Luo et al. ${ }^{17}$ 1998). É sabido que as forças da mastigação ocorrem em vários sentidos e direções, e, portanto, ocorre também obliquamente em relação ao plano oclusal, situação essa que não foi simulada neste estudo. Consequentemente, fica difícil reproduzir todos os detalhes do comportamento natural. De qualquer forma, diante de todas essas limitações, os níveis e locais de tensões gerados mostram as diferenças e similaridades da concentração de carga em overdenture retida sobre implantes, quando estes apresentam duas angulações diferentes, por meio do sistema de retenção barra-clipe.

\section{CONCLUSÃO}

Considerando-se as limitações deste estudo in vitro e de acordo com a metodologia empregada, concluiu-se que não houve semelhança nos campos de tensão quando comparados os modelos de análise fotoelástica AFIP e AFII. Isso porque o modelo fotoelástico com implantes paralelos apresentou uma maior concentração de tensão no ápice dos implantes, ao passo que o modelo fotoelástico com implantes angulados uma maior concentração de tensão na mesial dos implantes.
PIGOZZOMN, LAGANÁ DC, NORItOME PY. AVALIAÇÃO DAS tensões às ESTRUTURAS DE SUPORTE, POR MEIO DO MÉTODO FOTOELÁSTICO, DECORRENTES DAS FORÇAS APLICADAS SOBRE OVERDENTURES RETIDAS EM IMPLANTES COM SISTEMA DE ENCAIXE BARRACLIPE

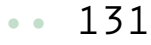

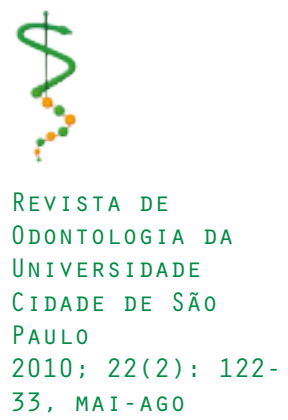


IGOZZO $M N$,

LAGANÁ DC,

NORITOME PY.

AVALIAÇÃO DAS

TENSÕES ÀS

ESTRUTURAS DE

SUPORTE, POR

MEIO DO MÉTODO

FOTOELÁSTICO,

DECORRENTES

DAS FORÇAS

APLICADAS SOBRE

OVERDENTURES

RETIDAS EM

IMPLANTES COM

SISTEMA DE

ENCAIXE BARRA -

CLIPE

132

\section{REFERÊNCIAS}

1. Rutkunas V, Mizutani H, Peciuliene V, Bendinskaite R, Linkevicius T.Maxillary complete denture outcome with two-implant supported mandibular overdentures. A systematic review. Stomatogija 2008; 10(01): 10-5.

2. Naert I, Quirynen M, Hooghe M, van Steenberghe D. A comparative prospective study of splinted and unsplintes Bránemark implants in mandibular overdenture therapy: a preliminary report. J Prosthet Dent. 1994 May; 71(5): 486-90.

3. Ochiai KT, Williams BH, Hojo S, Nishimura R, Caputo AA. Photoelastic analysis of the effect of palatal support on various implant-supported overdenture designs. J Prosthet Dent 2004 May; 91(5): 421-7.

4. Rentsch-Kollar A, Huber S, Mericske-Stern R. Mandibular implant overdentures followed for over 10 years: patient compliance and prosthetic maintenance. Int J Prosthodont 2010 Mar-Apr;23(2):91-8.

5. Naert I, Gizani S, van Steenberghe D. Bone behavior around sleeping and nonsleeping implants retaining a mandibular hinging overdenture. Clin Oral Implants Res. 1999 Apr; 10(2): 149-54.

6. Walton JN, MacEntee MI, Glick N. One-year prosthetic outcomes with implants overdentures; a randomized clinical trial. Int J Oral maxillofac Implants. 2002 MayJun; 17(3): 391-8.

7. Thayer HH, Caputo AA. Photoelastic stress analysis of Overdenture attachments. J Prosthet Dent 1980 Jun; 43(6): 611-617.

8. Federick DR, Caputo AA. Effects of Overdenture retention designs and implant orientation on load transfer characteristics. J Prosthet Dent 1996; 76(6): 624-631.

9. Kenney R, Richards MW. Photoelastic stress patterns produced by implant-retained Overdentures. J Prosthet Dent 1998 Nov; 80(5): 559-64.

10. Sadowsky SJ, Caputo AA. Effect of anchorage system and extension base contact on load transfer with mandibular implant-retained Overdenture. J Prosthet Dent 2000 Sep; 84(3): 327-34.

11. Thayer HH, Caputo AA. Occlusal force transmission by overdenture attachments. J Prosthet Dent 1979 Mar; 41(3): 266-71.

12. Porter AJ, Petropoulos VC, Brunski JB. Comparison of load distribution for implant overdenture attachment. Int J Oral Maxillofac Implants 2002; 17(5): 651-662.

13. Gonini Jr A. Análise fotoelástica da tensão oclusal produzida por uma sobredentadura implanto-retida com base de material resiliente [Tese].São Paulo: Faculdade de Odontologia da USP; 2002.

14. Gomes F. Análise fotoelástica de dissipação de cargas oclusais através de overdentures mandibulares com barra dolder resiliente implanto retida. [Tese]. São Paulo: Faculdade de Odontologia da USP; 2005.

15. Ichikawa T, Horiuchi M, Wigianto R, Matsumoto N. In vitro study of mandibular implant-retained overdenture: The influence of stud attachments on load transfer to the implant and the soft tissue. Int J Prosthodont 1996 Jul-Aug; 9(4): 394-9.

16. Covaciuc Y. Análise fotoelástica da distribuição de tensões sobre as estruturas desuporte de próteses totais, convencional e overdenture implanto-suportada retida por O'ring, contendo diferentes tipos de dentes artificiais. [Mestrado] Faculdade de Odontologia da USP; 2002. 
17. Luo X, Ouyang G, Ma X. Three dimensional finite element analysis on the mandibular complete overdenture supported by nature roots or implants. Zhonghua Kou Qiang Yi Xue Za Zhi. 1998 Sep; 33(5): 303-5.

18. Bortoli Jr N. Análise fotoelástica de distribuição de tensões sobre overdentures e implantes, com o uso de barra-clipe ou attachment bola. [Tese]. São Paulo: Faculdade de Odontologia da USP; 2004.

19. Piagge CSLD. Análise fotoelástica do rebordo sob uma overdenture implantosuportada,associada à barra de estabilização, encaixe ERA e magneto [Tese]. São Paulo: Faculdade de Odontologia da USP; 2002.

20. Silva DP, Cazal C, Almeida FC, Dias RB, Ballester RY. Photoelastic stress analysis surrounding implant-supported prosthesis and alveolar ridge on mandibular overdentures. Int J Dent 2010;2010:780670.

21. Menicucci G, Lorenzetti M, Pera P, Preti G. Mandibular implant-retained overdenture: a clinical trial of two anchorage systems. Int J Oral Maxillofac Implants 1998 Nov-Dec; 13(6): 851-856.

22. Celik G, Uludag B. Photolelastic stress analysis of various retention mechanisms on 3-implant-retained mandibular overdentures. J Prosthet Dent 2007 Apr; 97(4):22935.

23. Eser A, Akça K, Eckert S, Cehreli MC. Nonlinear finite element analysis versus ex vivo strain gauge measurements on immediately loaded implants. Int J Oral Maxillofac Implants. 2009 May-Jun; 24(3):439-46.

Recebido em: 31/05/2010

Aceito em: 16/03/2010
PIGOZZO MN,

LAGaná DC,

NORITOME PY.

AVALIAÇÃO DAS

TENSÕES ÀS

ESTRUTURAS DE

SUPORTE, POR

meio do método

FOTOELÁSTICO,

DECORRENTES

DAS FORÇAS

APLICADAS SOBRE

oVERDENTURES

RETIDAS EM

IMPLANTES COM

SISTEMA DE

ENCAIXE BARRA-

$C L I P E$

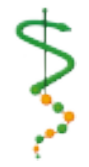

REVISTA DE

ODONTOLOGIA DA

UN I VERS I DADE

CIDADE DE SÃO

PAULO

$2010 ; 22(2): 122$ -

33, MAI-AGO 